\title{
A Retrospective Analysis of Thromboembolic Phenomena in Mechanically Ventilated Patients with COVID-19
}

\author{
Fahad Faqihi, ${ }^{1}$ Abdulrahman Alharthy, ${ }^{1}$ Abdullah Balhamar, ${ }^{1}$ Nasir Nasim, ${ }^{1}$ \\ Khaled Alanezi, ${ }^{2}$ Feisal Alaklobi, ${ }^{2}$ Ziad A. Memish, ${ }^{3}$ Mike Blaivas, ${ }^{4}$ Saleh A. Alqahtani, ${ }^{5}$ \\ and Dimitrios Karakitsos $\mathbb{D}^{1,4}$ \\ ${ }^{1}$ Critical Care Department, King Saud Medical City, Riyadh, Saudi Arabia \\ ${ }^{2}$ King Saud Medical City, Riyadh, Saudi Arabia \\ ${ }^{3}$ Research and Innovation Center, King Saud Medical City, Riyadh, Saudi Arabia \\ ${ }^{4}$ Department of Internal Medicine, University of South Carolina, School of Medicine, Columbia, SC, USA \\ ${ }^{5}$ Transplant Hepatology Division of Gastroenterology and Hepatology, Johns Hopkins University, School of Medicine, Baltimore, \\ MD, USA
}

Correspondence should be addressed to Dimitrios Karakitsos; karakitsosdimitrios@gmail.com

Received 19 July 2020; Revised 27 November 2020; Accepted 30 December 2020; Published 13 January 2021

Academic Editor: Fred A. Luchette

Copyright (c) 2021 Fahad Faqihi et al. This is an open access article distributed under the Creative Commons Attribution License, which permits unrestricted use, distribution, and reproduction in any medium, provided the original work is properly cited.

Background. Recent studies have shown an increased prevalence of thromboembolic disease in critically ill patients with the novel SARS-CoV-2 disease (COVID-19). However, the use of enhanced anticoagulation therapy in these patients remains controversial. Objectives. To determine the incidence of thromboembolic phenomena (TEP) and hemorrhagic events (HEs) in intensive care unit (ICU) COVID-19 patients. Methods. One hundred and sixty ICU patients with COVID-19 were enrolled. Clinical examination results, laboratory data, and imaging studies (computed tomography/Doppler ultrasound scans) for these patients were retrospectively collected and analyzed. Outcome measures including days on mechanical ventilation, ICU length of stay, and day- 28 mortality were recorded. Results. Sixty patients (37.5\%) developed TEP including thirty patients with deep vein thrombosis, 55 patients with pulmonary embolism, and 7 patients with arterial thromboembolism. Cardiac arrhythmias, lymphocytopenia, and increased D-dimers were more frequently observed in the TEP group compared to the non-TEP group of patients (all $p<0.05)$. The sensitivity, specificity, and positive and negative predictive values of a cutoff D-dimer level of $3.0 \mu \mathrm{g} / \mathrm{mL}$ for predicting PE were $74.5 \%, 95.1 \%, 86.8 \%$, and $91.9 \%$, respectively. Thirteen patients experienced HEs, which were more frequently observed in the TEP group $(p<0.05)$. Twenty-eight-day mortality was higher in the TEP group $(60 \%)$ compared to the non-TEP group (30\%) of patients $(p=0.02)$. Conclusions. The rates of TEP and HEs in mechanically ventilated critically ill COVID-19 patients were $37.5 \%$ and $8.1 \%$. Twenty-eight-day mortality was higher in the TEP group (60\%) compared to the non-TEP group (30\%) of patients.

\section{Introduction}

The novel coronavirus SARS-CoV-2 disease (COVID-19) emerged in Wuhan city, the capital of Hubei Province in China, and has progressively spread throughout the world. A minority of patients can develop life-threatening disease, which is characterized by acute respiratory distress syndrome (ARDS), sepsis, multisystem organ failure, neurological and other extrapulmonary manifestations, and thromboembolic disease [1].
An increased prevalence of pulmonary embolism (PE) in critically ill COVID-19 patients was previously reported $[2,3]$. Also, the administration of enhanced anticoagulation in patients with severe COVID-19 and Padua prediction score $\geq 4$ or D-dimers $>3.0 \mu \mathrm{g} / \mathrm{mL}$ was suggested [4]. Recently, we documented a PE rate of approximately $25 \%$ in a point-of-care ultrasound study in critically ill COVID-19 patients [5]. Herein, we present a brief retrospective analysis of thromboembolic phenomena (TEP) in mechanically ventilated critically ill COVID-19 patients. 


\section{Methods}

We retrospectively analyzed COVID-19 patients that were admitted to our 300-bed intensive care unit (ICU) between April 1 and May 30, 2020. All patients were diagnosed according to the World Health Organization guidelines [6]. COVID-19 was confirmed by real-time polymerase chain reaction (RT-PCR) assays, using QuantiNova Probe RTPCR kit (Qiagen, GmbH, Germany) in a light-cycler 480 real-time PCR system (Roche, Basel, Switzerland), which were performed on nasopharyngeal swabs [7]. The coagulation profiles of patients, which included prothrombin time, activated partial thromboplastin time, thrombin time, international normalized ratio, fibrinogen, and D-dimers, were performed using an automatic coagulation analyzer (Sysmex CS-2500 System, Siemens, Germany) and were retrieved from their electronic medical record files. Upon ICU admission, the Sequential Organ Function Assessment (SOFA) and the Acute Physiology and Chronic Health Evaluation II (APACHE II) scores were included in the analysis. Researchers recorded all TEP during the patients' ICU length of stay such as PE, deep vein thrombosis, and arterial thromboembolism based on clinical examinations, chest computed tomography (CT), CT angiography, and lower limb venous Doppler ultrasound. Hemorrhagic events (HEs) defined as pause of anticoagulation therapy with blood product transfusion and surgical intervention/death were registered. Outcome measures such as days on mechanical ventilation, ICU length of stay, and 28-day mortality were included in the analysis. All data were retrieved from the patients' records and were retrospectively analyzed. The study was conducted according to the principles outlined in the Declaration of Helsinki and was approved by our Institutional Review Board.

\section{Statistical Analysis}

Continuous variables are expressed as medians with interquartile ranges (IQRs) and categorical variables as absolute numbers/ratios. We utilized the Wilcoxon signed rank test for nonparametric data for group comparisons. All tests were two-tailed and considered significant when the $p$ value was $<0.05$. Analysis was performed using SPSS, version 23.0.

\section{Results}

Of the total two hundred and twenty ICU patients that were admitted during the study period, only one hundred and sixty were included in the final analysis. Sixty patients were excluded as they were transferred to other COVID-19 centers according to our Ministry of Health surge plan. The main characteristics of these one hundred and sixty patients are illustrated in Table 1. The vast majority of our patients were males (125 out of 160) and relatively young as their median age was 49 (IQR: 39-58). Upon ICU admission, all patients had increased SOFA and APACHE II scores. All patients had ARDS and were mechanically ventilated (Table 1). We administered ARDS-net/prone positioning ventilation and empiric treatment with ribavirin, interferon beta- $1 \mathrm{~b}$, antibiotics, and ICU supportive care to all patients [8]. All mechanically ventilated COVID-19 patients received intermittent pneumatic compression and baseline weightand renal function-adjusted doses of low-molecular-weight heparin thromboprophylaxis unless contraindicated (enoxaparin $20 \mathrm{mg}$ once daily if $<50 \mathrm{~kg}$, enoxaparin $40 \mathrm{mg}$ once daily if $50-100 \mathrm{~kg}, 40 \mathrm{mg}$ twice daily if $101-150 \mathrm{~kg}$, and $60 \mathrm{mg}$ twice daily if $>150 \mathrm{~kg}$ ) as per hospital protocol [8]. Upon ICU admission (baseline), no hemorrhagic events were recorded; however, 8 out of 160 patients had a history of thromboembolic disease (Table 1).

During hospitalization, sixty patients (37.5\%) developed TEP including thirty patients with deep vein thrombosis, fifty-five patients with $\mathrm{PE}$, and seven patients with arterial thromboembolism. These patients received treatment-dose systemic anticoagulation based on the individual case scenario and as per hospital protocol [8]. None of the patients had evidence of developing diffuse intravascular coagulopathy (data not included in Table 1). Specifically, platelet count, prothrombin time, activated partial thromboplastin time, thrombin time, and international normalized ratio did not significantly differ during the study period. Also, no other systemic disorders (i.e., autoimmune disorders) and/ or thrombophilia were detected. Parameters that were more frequently observed in the TEP group versus the non-TEP group were as follows: history of cardiac arrhythmias, lymphocytopenia, and increased levels of D-dimer (all $p<0.05$, Table 1). PE was confirmed by contrast chest CT, which revealed five patients with massive $\mathrm{PE}$, forty patients with subsegmental $\mathrm{PE}$, and ten patients with pulmonary vascular microthrombosis. Using a previously suggested cutoff D-dimer value of $3.0 \mu \mathrm{g} / \mathrm{mL}$ for predicting $\mathrm{PE}$, our analysis revealed that sensitivity, specificity, and positive and negative predictive values were $74.5 \%, 95.1 \%, 86.8 \%$, and $91.9 \%$, respectively. In the TEP group, after the administration of anticoagulation therapy, D-dimers gradually decreased over a period of two weeks (from 5.1 (IQR: 2.2-9.1) to 1.6 (IQR: 0.7-1.9); $p=0.001$, Wilcoxon signed rank test)). Mortality on day 28 was higher in the TEP group $(60 \%)$ compared to the non-TEP group (30\%) of patients $(p=0.02$, Table 1).

Thirteen patients developed hemorrhagic events, which were more frequently observed in patients with TEP receiving therapeutic anticoagulation $(p<0.05$, Table 1). The characteristics of these thirteen patients with hemorrhagic events are illustrated in Table 2. Anticoagulation was interrupted in all cases. Major hemorrhagic events requiring transfusion of blood products were observed in the TEP group of patients. Seven out of thirteen cases required transfusion of blood products, while in four cases, massive transfusion protocol (transfusion of 4 units of red blood cells in less than 4 hours, plus hemodynamic instability and ongoing bleeding) was activated (Table 2). However, anticoagulation was resumed in seven patients approximately 5 (IQR: 2-7) days after the HE. Serial ultrasound surveillance for detection of deep vein thrombosis was applied on all cases. Four patients of the TEP group 
TABLE 1: Characteristics of critically ill patients with COVID-19 who developed ( $n=60,37.5 \%)$ or not $(n=100,62.5 \%)$ thromboembolic phenomena.

\begin{tabular}{|c|c|c|c|c|}
\hline Parameters & $\begin{array}{l}\text { All patients, } \\
\quad n=160\end{array}$ & $\begin{array}{c}\text { Patients without TEP, } n=100 \\
(62.5 \%)\end{array}$ & $\begin{array}{l}\text { Patients with TEP, } n=60 \\
(37.5 \%)\end{array}$ & $p$ value \\
\hline Age (years) & $49(39-58)$ & $49(38-59)$ & $50(39-58)$ & 0.9 \\
\hline Sex (males/females) & $125 / 35$ & $80 / 20$ & $45 / 15$ & 0.6 \\
\hline Body mass index $\left(\mathrm{kg} / \mathrm{m}^{2}\right)$ & $27(21-32)$ & $27(20-32)$ & $28(20-32)$ & 0.45 \\
\hline Baseline SOFA score & $6(4-10)$ & $6(3-9)$ & $7(4-11)$ & 0.25 \\
\hline Baseline APACHE II score & $19(16-23)$ & $18(16-22)$ & $19(17-23)$ & 0.19 \\
\hline \multicolumn{5}{|l|}{ Comorbidities } \\
\hline Hypertension (yes/no) & $100 / 60$ & $60 / 40$ & $40 / 20$ & 0.6 \\
\hline Diabetes mellitus (yes/no) & $70 / 90$ & $40 / 60$ & $30 / 30$ & 0.5 \\
\hline Cardiovascular disease (yes/no) & $30 / 130$ & $20 / 80$ & $10 / 50$ & 0.45 \\
\hline Cardiac arrhythmias (yes/no) & $16 / 144$ & $4 / 96$ & $12 / 48$ & $0.04^{*}$ \\
\hline Thromboembolic disease (yes/no) & $8 / 152$ & $5 / 95$ & $3 / 57$ & 0.5 \\
\hline Chronic respiratory disorder (yes/no) & $18 / 142$ & $10 / 90$ & $8 / 52$ & 0.45 \\
\hline End-stage kidney disease (yes/no) & $14 / 146$ & $8 / 92$ & $6 / 54$ & 0.5 \\
\hline \multicolumn{5}{|l|}{ Baseline hematological profile } \\
\hline Platelets (G/L) & $229(207-291)$ & $228(213-256)$ & $231(207-268)$ & 0.56 \\
\hline Fibrinogen $(\mathrm{g} / \mathrm{L})$ & $7.5(5.3-9)$ & $7.7(5.5-8.9)$ & $7.6(5.2-8.7)$ & 0.35 \\
\hline D-Dimers $(\mu \mathrm{g} / \mathrm{ml})$ & $2.7(1.2-8.9)$ & $2.8(1.2-5.5)$ & $5.1(2.2-9.1)$ & $0.02^{*}$ \\
\hline White blood cells $\left(10^{9} / \mathrm{L}\right)$ & $20(16-24)$ & $19(14-23)$ & $19(16-24)$ & 0.75 \\
\hline Lymphocytes $\left(10^{9} / \mathrm{L}\right)$ & $0.99(0.71-1.2)$ & $0.98(0.77$ to 1.2$)$ & $0.75(0.45$ to 1.1$)$ & $0.035^{*}$ \\
\hline \multicolumn{5}{|l|}{ Anticoagulation therapy } \\
\hline Prophylactic anticoagulation & $160 / 160$ & $100 / 100$ & $60 / 60$ & 0.9 \\
\hline Therapeutic anticoagulation & $60 / 160$ & $0 / 100$ & $60 / 60$ & $0.001^{*}$ \\
\hline \multicolumn{5}{|l|}{ Thromboembolic phenomena } \\
\hline Deep vein thrombosis (yes/no) & $30 / 130$ & $0 / 100$ & $30 / 30$ & $0.001^{*}$ \\
\hline Pulmonary thromboembolism (yes/no) & $55 / 105$ & $0 / 100$ & $55 / 5$ & $0.001^{*}$ \\
\hline Arterial thromboembolism (yes/no) & $7 / 153$ & $0 / 100$ & $7 / 53$ & $0.04^{*}$ \\
\hline Hemorrhagic events (yes/no) & $13 / 147$ & $3 / 97$ & $10 / 50$ & $0.045^{*}$ \\
\hline \multicolumn{5}{|l|}{ Outcome measures } \\
\hline Days on mechanical ventilation & $18(14-26)$ & $18(12-25)$ & $20(14-29)$ & 0.35 \\
\hline ICU length of stay & $21(16-28)$ & $20(16-26)$ & $21(17-30)$ & 0.35 \\
\hline Extracorporeal membrane oxygenation & $7 / 153$ & $4 / 96$ & $3 / 57$ & 0.6 \\
\hline Continuous renal replacement therapy & $19 / 141$ & $10 / 90$ & $9 / 51$ & 0.35 \\
\hline $\begin{array}{l}\text { Mortality on day } 28 \text { post-ICU admission } \\
(n, \%)\end{array}$ & $54(33.75 \%)$ & $30(30 \%)$ & $24(60 \%)$ & $0.02^{*}$ \\
\hline
\end{tabular}

$\mathrm{TEP}=$ thromboembolic phenomena; ICU = intensive care unit; APACHE II score= Acute Physiology and Chronic Health Evaluation II score; SOFA score $=$ Sequential Organ Function Assessment score. Values are medians with interquartile ranges. ${ }^{*} p$ values $<0.05$ were statistically significant (Wilcoxon signed rank test).

underwent inferior vena cava filter placement. Finally, two out of the thirteen cases (TEP group) were fatal due to intracranial hemorrhage.

\section{Discussion}

This retrospective study has several limitations, which prevent its generalizability. However, we confirmed the increased incidence of TEP in mechanically ventilated critically ill COVID-19 patients as other studies previously reported [2-4]. Of note, we found a higher rate of cardiac arrhythmias, lymphocytopenia, and increased D-dimers in patients with TEP $[1,9,10]$. Hypercoagulability with associated vascular dysfunction and ensuing cytokine release syndrome might be the underlying mechanism promoting thromboinflammation in complex COVID-19 critical illness [9-12]. Also, the fact that SARS-CoV-2 can bind to the ACE2 receptors that are expressed on type II pneumocytes and vascular endothelial cells within the lung could reflect direct injury to the pulmonary vasculature [13]. This vascular pathology along with the parenchymal lung injury ("dualhit" underlying mechanism) could be at least partially responsible for the refractory ARDS observed in critically ill patients with COVID-19 [14].

In this study (April to May 2020), the increased observed prevalence of $\mathrm{PE}$ as compared to our previously reported data (lower PE rate) for the month of June 2020 could be explained by the fact that the retrospective data were derived from the early stages of the COVID-19 outbreak in our country [5]. Hence, the observed discrepancies could reflect the change in our practices such as the integration of a more robust anticoagulation protocol for critically ill COVID-19 patients and better surveillance for the development of TEP $[5,8]$.

We found that an elevated D-dimer level $>3.0 \mu \mathrm{g} / \mathrm{mL}$ could predict the development of PE in mechanically 
TABLE 2: Characteristics of thirteen critically ill COVID-19 patients who developed hemorrhagic events.

\begin{tabular}{lcc}
\hline Parameters & Patients without TEP, $n=3$ & Patients with TEP, $n=10$ \\
\hline Age (years) & $55(53-57)$ & $56(54-60)$ \\
Sex $($ males/females) & $1 / 2$ & $6 / 4$ \\
Body mass index $\left(\mathrm{kg} / \mathrm{m}^{2}\right)$ & $29(27-31)$ & $30(28-32)$ \\
Baseline SOFA score & $7(6-9)$ & $8(7-10)$ \\
Baseline APACHE II score & $20(19-21)$ & $21(19-23)$ \\
Comorbidities & \\
Hypertension (yes/no) & $3 / 0$ \\
Diabetes mellitus (yes/no) & $1 / 2$ \\
Cardiovascular disease (yes/no) & $1 / 2$ \\
Cardiac arrhythmias (yes/no) & $1 / 2$ \\
Thromboembolic disease (yes/no) & - & $8 / 2$ \\
Chronic respiratory disorder (yes/no) & - & $5 / 5$ \\
End-stage kidney disease (yes/no) & - & $5 / 5$ \\
Hemorrhagic events and management & & $5 / 5$ \\
Minor hemorrhagic events (no transfusion, $n)$ & $2 / 8$ \\
Major hemorrhagic events (transfusion required, $n)$ & - \\
Anticoagulation interrupted $(n)$ & $3 / 6$ \\
Gastrointestinal bleeding requiring endoscopy $(n)$ & - & $2 / 8$ \\
Intracranial hemorrhage $(n)$ & - & 3 \\
Massive transfusion protocol activated $(n) *$ & - & 7 \\
Inferior vena cava filter placed $(n)$ & - & 7 \\
Serial ultrasound surveillance for DVT $(n)$ & 3 \\
Fatalities $(n)$ & - & 2 \\
\hline
\end{tabular}

TEP = thromboembolic phenomena; ICU = intensive care unit; APACHE II score= Acute Physiology and Chronic Health Evaluation II score; SOFA score $=$ Sequential Organ Function Assessment score; DVT: deep vein thrombosis. ${ }^{*}$ Massive transfusion was defined as replacement of $>1$ blood volume in 24 hours or $>50 \%$ of blood volume in 4 hours (adult blood volume is approximately $70 \mathrm{~mL} / \mathrm{kg}$ ).

ventilated COVID-19 patients with a sensitivity of $74.5 \%$ and a specificity of $95.1 \%$. Moreover, D-dimer levels gradually decreased after the administration of anticoagulation therapy. Hence, upgrading the systemic anticoagulation in mechanically ventilated patients with life-threatening COVID-19 may be a step in the right direction [4]. However, patients receiving enhanced therapeutic anticoagulation could develop major hemorrhagic events, and therefore, close ICU monitoring is warranted as the risk is not negligible. In this study, a more meaningful subgroup analysis of patients who developed hemorrhagic events was not feasible due to the small number of cases. We speculate that given the absence of such events upon ICU admission (baseline), their development was linked to the administration of therapeutic anticoagulation although the presence of COVID-19-associated coagulopathy cannot be excluded.

In this series, COVID-19-associated coagulopathy was not clearly recorded; however, the latter may exhibit overlapping features of hemophagocytic syndrome, antiphospholipid antibodies, and thrombotic microangiopathy [15-17]. The presence of severe coagulopathy may complicate the administration of therapeutic anticoagulation in critically ill COVID-19 patients although thromboembolic disease per se appears to be more common in COVID-19 compared to sepsis-induced coagulopathy. Despite the aforementioned considerations, the increased mortality rate in mechanically ventilated COVID-19 patients with TEP was consistent with recently published data [18].

\section{Conclusion}

We found that the rate of thromboembolic phenomena and hemorrhagic events in critically ill patients with COVID-19 was $37.5 \%$ and $8.1 \%$, respectively. D-Dimer level can be used in identifying high risk patients for developing TEP. Finally, the optimal anticoagulation regime [19], the interactions between anticoagulants and antivirals, and the follow-up prophylaxis with new oral anticoagulants [20] based on risk stratification algorithms could be explored in future prospective studies.

\section{Abbreviations}

$\begin{array}{ll}\text { SARS-CoV-2 } & \text { COVID-19 } \\ \text { disease: } & \\ \text { TEP: } & \text { Thromboembolic phenomena } \\ \text { HE: } & \text { Hemorrhagic events } \\ \text { ICU: } & \text { Intensive care unit } \\ \text { CT: } & \text { Computed tomography } \\ \text { PE: } & \text { Pulmonary embolism }\end{array}$

SOFA score: Sequential Organ Function Assessment score 


\author{
APACHE II Acute Physiology and Chronic Health \\ score: $\quad$ Evaluation II score \\ ARDS: $\quad$ Acute respiratory distress syndrome.
}

\section{Data Availability}

All pertinent data sets included in this study are available from the corresponding author on reasonable request.

\section{Additional Points}

Highlights. We observed that the rates of TEP and HEs in mechanically ventilated critically ill COVID-19 patients were $37.5 \%$ and $8.1 \%$. Twenty-eight-day mortality was higher in the TEP group (60\%) compared to the non-TEP group $(30 \%)$ of patients. The sensitivity, specificity, and positive and negative predictive values of a cutoff D-dimer level of $3.0 \mu \mathrm{g} / \mathrm{mL}$ for predicting pulmonary embolism in critically ill COVID-19 patients were 74.5\%, 95.1\%, 86.8\%, and $91.9 \%$, respectively. Enhanced anticoagulation in critically ill COVID-19 patients with Padua prediction score $\geq 4$ or D-dimers $>3.0 \mu \mathrm{g} / \mathrm{mL}$ may be warranted; however, the administration of enhanced therapeutic anticoagulation requires close monitoring as the risk for hemorrhage is not negligible.

\section{Ethical Approval}

This study was approved by the Institutional Review Board of King Saud Medical City, Riyadh, Kingdom of Saudi Arabia (H-01-R-053, IORG0010374\#, H1RI-16-20-02).

\section{Consent}

All participants or their legal representatives provided informed consent.

\section{Conflicts of Interest}

FF, AA, AB, NN, KA, FA, ZAM, SAA, and DK declare that there are no conflicts of interest. $\mathrm{MB}$ consults for EthosMedical, 410Medical, EchoNous, and Sonosim; none of these companies were aware of the study or had influence on it.

\section{Authors' Contributions}

$\mathrm{FF}, \mathrm{AA}, \mathrm{AB}, \mathrm{NN}, \mathrm{KA}, \mathrm{FA}$, and SAA collected the data, performed the statistical analysis, and drafted the initial version of the manuscript. ZAM, MB, and DK designed the study and drafted the final version of the manuscript. All authors reviewed the final version of the manuscript and approved its submission to the journal.

\section{Acknowledgments}

The authors acknowledge all healthcare workers in the global fight against COVID-19.

\section{References}

[1] G. Grasselli, A. Zangrillo, A. Zanella et al., "Baseline characteristics and outcomes of 1591 patients infected with SARSCoV-2 admitted to ICUs of the lombardy region, Italy," JAMA, vol. 323, no. 16, pp. 1574-1581, 2020.

[2] S. Cui, S. Chen, X. Li, S. Liu, and F. Wang, "Prevalence of venous thromboembolism in patients with severe novel coronavirus pneumonia," Journal of Thrombosis and Haemostasis, vol. 18, no. 6, pp. 1421-1424, 2020.

[3] F. A. Klok, M. J. H. A. Kruip, N. J. M. van der Meer et al., "Incidence of thrombotic complications in critically ill ICU patients with COVID-19," Thrombosis Research, vol. 191, pp. 145-147, 2020.

[4] N. Tang, H. Bai, X. Chen, J. Gong, D. Li, and Z. Sun, “Anticoagulant treatment is associated with decreased mortality in severe coronavirus disease 2019 patients with coagulopathy," Journal of Thrombosis and Haemostasis, vol. 18, no. 5, pp. 1094-1099, 2020.

[5] A. Alharthy, F. Faqihi, M. Abuhamdah et al., "Prospective longitudinal evaluation of point-of-care lung ultrasound in critically ill patients with severe COVID-19 pneumonia," Journal of Ultrasound in Medicine, In press.

[6] WHO, "Laboratory testing for 2019 novel coronavirus (2019$\mathrm{nCoV}$ ) in suspected human cases," Interim Guidance, https:// www.who.int/publications-detail/laboratory-testing-for-2019novel-coronavirus-in-suspectedhuman-cases-20200117, 2020.

[7] J. F. Chan, C. C. Yip, K. K. To et al., "Improved molecular diagnosis of COVID-19 by the novel, highly sensitive and specific COVID-19-RdRp/Hel real-time reverse transcription-PCR assay validated in vitro and with clinical specimens," Journal of Clinical Microbiology, vol. 58, no. 5, 2020.

[8] Saudi Ministry of Health, "Coronavirus diseases 19 (COVID19) guidelines," 2020, https://covid19.moh.gov.sa.

[9] M. Fraissé, E. Logre, O. Pajot, H. Mentec, G. Plantefève, and D. Contou, "Thrombotic and hemorrhagic events in critically ill COVID-19 patients: a French monocenter retrospective study," Critical Care, vol. 24, no. 1, p. 245, 2020.

[10] W. J. Guan, Z. Y. Ni, Y. Hu et al., "China medical treatment expert group for covid-19 clinical characteristics of coronavirus disease 2019 in China," New England Journal of Medicine, vol. 382, no. 18, pp. 1708-1720, 2020.

[11] F. Zhou, T. Yu, R. Du et al., "Clinical course and risk factors for mortality of adult inpatients with COVID-19 in Wuhan, China: a retrospective cohort study," The Lancet, vol. 395, no. 10229, pp. 1054-1062, 2020.

[12] M. Panigada, N. Bottino, P. Tagliabue, G. Grasselli et al., "Hypercoagulability of COVID-19 patients in intensive care unit. a report of thromboelastography findings and other parameters of hemostasis," Journal of Thrombosis and Haemostasis, vol. 18, no. 7, 2020.

[13] Z. Varga, A. J. Flammer, P. Steiger et al., "Endothelial cell infection and endotheliitis in COVID-19," The Lancet, vol. 395, no. 10234, pp. 1417-1418, 2020.

[14] A. Alharthy, F. Faqihi, Z. A. Memish, and D. Karakitsos, "Lung injury in COVID-19-an emerging hypothesis," ACS Chemical Neuroscience, vol. 11, no. 15, pp. 2156-2158, 2020.

[15] H. Mei and H. Yu, "Characteristics, causes, diagnosis and treatment of coagulation dysfunction in patients with COVID-19," Zhonghua Xue Ye Xue Za Zhi, vol. 41, no. 3, p. $185,2020$.

[16] T. Iba, J. H. Levy, J. M. Connors, T. E. Warkentin, J. Thachil, and M. Levi, "The unique characteristics of COVID-19 coagulopathy," Critical Care, vol. 24, no. 1, p. 360, 2020. 
[17] A. Warkentin, F. Faqihi, A. Balhamar, Z. A. Memish, and D. Karakitsos, "Life-threatening COVID-19 presenting as stroke with antiphospholipid antibodies and low ADAMTS13 activity, and the role of therapeutic plasma exchange: a case series," SAGE Open Medical Case Reports, vol. 8, p. 2050313X2096408, 2020.

[18] I. Paranjpe, V. Fuster, A. Lala et al., "Association of treatment dose anticoagulation with in-Hospital survival among hospitalized patients with COVID-19," Journal of the American College of Cardiology, vol. 76, no. 1, pp. 123-124, 2020.

[19] J. Thachil, N. Tang, S. Gando et al., "ISTH interim guidance on recognition and management of coagulopathy in COVID-19," Journal of Thrombosis and Haemostasis, vol. 18, no. 5, pp. 1023-1026, 2020.

[20] A. Alharthy, A. Balhamar, F. Faqihi et al., "Insidious development of pulmonary embolism in asymptomatic patients with COVID-19: two rare case-reports," Respiratory Medicine Case Reports, vol. 31, p. 101186, 2020. 